\title{
NOVEL METHOD FOR COMPARISON OF PRE-PLANNED ABLATION LINES FOR TREATMENT OF ATRIAL FIBRILLATION USING A COMMON REFERENCE MODEL
}

\author{
Martin Koch ${ }^{\star} \quad$ Andreas Kleinoeder ${ }^{\star} \quad$ Felix Bourier ${ }^{\ddagger}$ \\ Joachim Hornegger ${ }^{\star} \quad$ Norbert Strobel ${ }^{\dagger}$ \\ * Pattern Recognition Lab, Friedrich-Alexander-Universität Erlangen-Nürnberg, \\ Erlangen, Germany \\ $\ddagger$ Klinik für Herzrhythmusstörungen, Krankenhaus Barmherzige Brüder, Regensburg, Germany \\ ${ }^{\dagger}$ Siemens AG, Healthcare Sector, Forchheim, Germany
}

\begin{abstract}
The standard approach in interventional treatment of atrial fibrillation (AFib) is pulmonary vein isolation (PVI). PVI can be achieved by placing radio-frequency (RF) lesions contiguously around the pulmonary veins attached to the left atrium. Since accurate lesion placement may be difficult, pre-planned ablation lines can be used for better navigation both when using mapping systems or also when relying on fluoro overlay techniques. By working with physicians in this field, we learned that clinically acceptable ablation lines are not necessarily limited to a unique line, but there appears to be some flexibility when defining regions within which ablation should be performed.

We present a novel method to investigate the dimensions of such a region based on comparing pre-planned ablation lines set up for different left atria. A common reference model is proposed as a means to combine and compare different pre-planned ablation lines. Based on our data, we found an average deviation of individually pre-planned ablation lines from their respective mean of $2.9 \pm 1.9 \mathrm{~mm}$ and $1.8 \pm 1.5 \mathrm{~mm}$ for right and left sided ipsilateral planning lines, respectively. Beyond ablation line assessment, this work also introduces a framework which can be extended to automatic pre-planning of ablation lines for PVI procedures.
\end{abstract}

Index Terms - atrial fibrillation, ablation line planning, shape modeling and analysis, pulmonary vein isolation,

\section{INTRODUCTION}

Since electrophysiology (EP) procedures involve fluoroscopic guidance, C-arm X-ray devices with image overlay functionality, fusing soft-tissue information with live fluoro images, offer an alternative navigation approach to mapping systems $[1,2]$. For EP procedures, these systems can be used to merge soft-tissue heart information derived from pre-procedurally acquired 3-D data sets as well as planning information with live fluoroscopy during the intervention [3]. A first approach for planning of atrial fibrillation has been presented by Hastenteufel et al. [4]. More recently, Brost et al. [5] proposed a system for interactive planning of EP procedures, especially atrial fibrillation ablation procedures involving cryo-balloons. An example of pre-planned ablation lines for PVI is shown in Figure 1. The use of pre-planned ablation lines is not restricted to fluoro overlay navigation. This kind of annotation can also be helpful when performing PVI using a mapping system, e.g., CARTO 3 (Biosense Webster, Diamong Bar, CA, USA) or Ensite Velocity (St. Jude Medical, St. Paul, MN, USA).

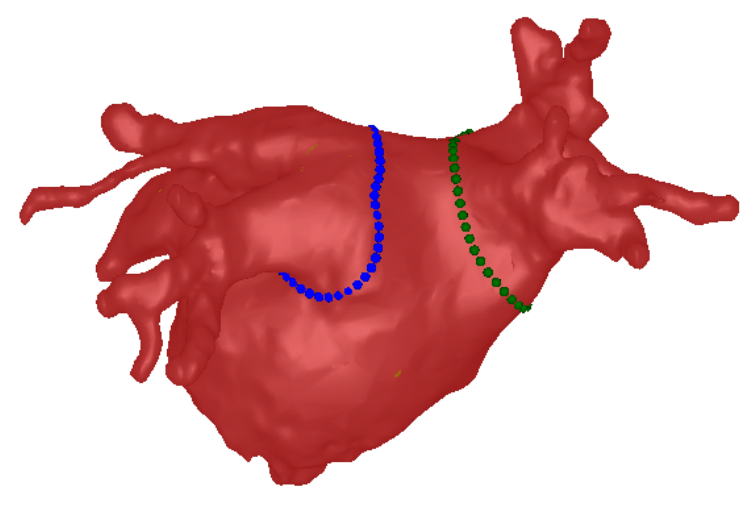

Fig. 1. Clinical left atrium surface model with pre-planned ipsilateral pulmonary vein (PV) ablation lines. The green line encircles the right PVs, the blue line the left PVs.

Pulmonary vein isolation is the treatment of choice for paroxysmal atrial fibrillation with a reported success rate of over $70 \%$ [6]. PVI is also applied to persistent AFib, however with lower success rate of just over $50 \%$ [6]. The structure of the left atrium is very complex. There are critical areas within the left atrium, e.g. the left atrial appendage or the area close to the esophagus, that should be avoided or treated with special care during ablation procedures. There are different strategies to perform PVI. Two common strategies are segmental ablation and ipsilateral pulmonary vein (PV) isolation. Arentz et al. investigated the influence of the isolation area around the PVI on the procedure outcome. Based on their findings, the ipsilateral ablation strategy is advantageous [7].

Personalized planning of ablation lines for electrophysiology procedures offers the potential to improve procedure success while lowering the risk to the patient. As of today, there has, however, not been an investigation on how ipsilateral ablation lines differ across similar cases. We present a novel method to investigate this problem by comparing pre-planned ablation lines defined by an experienced clinician. Although actual ablation lines may differ from their targets, a comparison among desirable ablation lines does provide insight into how much ablation lines may vary along certain parts of 


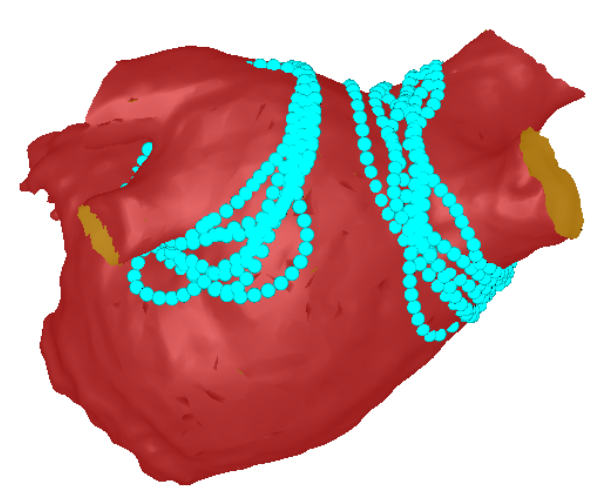

(a) Posterior view

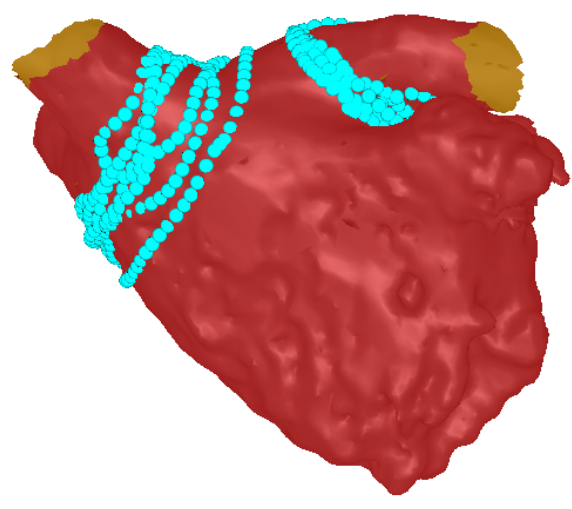

(b) Anterior view

Fig. 2. Estimated reference shape of the left atrium with pre-planned ablation lines transfered from seven different LA meshes. Blue lines represent the pre-planned ipsilateral PVI ablation lines. Compared to Figure 1, the PVs have been cut away $2 \mathrm{~cm}$ distal to the antrum to ensure better registration.

the left atrium.

\section{MATERIAL AND METHODS}

We used left atrial (LA) surface models of seven different patients to build up a common reference shape. The mesh models are represented as a triangulated mesh structure. The models cover the left atrium as well as a certain extent of the attached pulmonary veins. The length of the pulmonary veins varies from data set to data set. For consistency, a pre-processing step has been applied to all LA mesh models. The attached pulmonary veins have been removed from the mesh about $2 \mathrm{~cm}$ distal to the LA antrum.

The common reference shape is generated via pairwise non-rigid registration of the LA models. For registration, the mesh is seen as point cloud $\mathcal{M}$ consisting of $N$ points $\boldsymbol{x}_{i} \in \mathbb{R}^{3}$

$$
\mathcal{M} \equiv \boldsymbol{m}=\left[\boldsymbol{x}_{1}^{\top}, \ldots, \boldsymbol{x}_{N}^{\top}\right]^{\top} \in \mathbb{R}^{3 N}
$$

All meshes have the same anatomical orientation, and are zero centered before applying the registration.

Similar to our previous work [8], we used the Coherent Point Drift (CPD) algorithm [9] to perform the non-rigid point cloud registration. The main benefit of the CPD algorithm is the robustness against noise and outliers while generating smooth deformation fields. A pivot mesh $\boldsymbol{m}_{\text {pivot }}$ is registered to template meshes $\boldsymbol{m}_{t_{i}}$. The variables $t_{i}$ are used to refer to each of the $T=7$ template meshes.

Below, we describe how to derive a common reference model. Then we establish mean ablation lines. Afterwards, we evaluate how individual pre-planned ablation lines vary around their mean as we move around the left and right ipsilateral PVs.

\subsection{Non-Rigid Point Cloud Registration Using CPD}

In the first step, we selected one left atrium mesh model as pivot element,

$$
\boldsymbol{m}_{\text {pivot }}=\boldsymbol{m}_{t_{1}} \text {. }
$$

In the next step, we registered the pivot mesh to the remaining template meshes $\left\{\boldsymbol{m}_{t_{2}}, \ldots, \boldsymbol{m}_{t_{T}}\right\}$. We used the coherent point drift algorithm to compute the non-rigid transformation between the pivot mesh and the other left atrial mesh models. CPD follows a probabilistic approach by considering the alignment of the two point sets as a probability density estimation problem. The basic idea is to fit the Gaussian mixture model (GMM) centroids, represented by the points of the pivot mesh $\boldsymbol{m}_{\text {pivot }}$, to the template mesh $\boldsymbol{m}_{t_{i}}$, by maximizing the likelihood. This optimization is performed with the expectation maximization algorithm. During the optimization, the GMM centroids are forced to move coherently as a group, to ensure preservation of the topological structure of the point set.

The displacement function $v$ and the transformed point set $\tilde{\boldsymbol{m}}_{t_{i}}$ are obtained by minimizing the following energy function [9]:

$$
E\left(\tilde{\boldsymbol{m}}_{t_{i}}\right)=-\sum_{n=1}^{N} \log \sum_{m=1}^{M} e^{-\frac{1}{2}\left\|\frac{\boldsymbol{x}_{n}-\boldsymbol{y}_{m}}{\sigma}\right\|^{2}}+\frac{\lambda}{2} \phi(v),
$$

where $\phi(v)$ is a regularization to ensure the displacement field to be smooth. The parameter $\lambda$ determines the trade-off between data fitting and smoothness of the deformation field. We empirically determined a suitable value for this parameter $(\lambda=2.0)$.

\subsection{Common Reference Model}

For each template mesh $\boldsymbol{m}_{t_{i}}$ the estimated deformation field $\boldsymbol{u}_{t_{i}} \in$ $\mathbb{R}^{3 N}$ is calculated by registration of the pivot mesh $\boldsymbol{m}_{\text {pivot }}$ to $\boldsymbol{m}_{t_{i}}$. The transformed pivot mesh can then be described as

$$
\tilde{\boldsymbol{m}}_{t_{i}}=\boldsymbol{m}_{\mathrm{pivot}}+\boldsymbol{u}_{t_{i}}
$$

The common reference model is defined as the mean shape given by,

$$
\boldsymbol{m}_{\mathrm{ref}}=\frac{1}{T-1}\left(\sum_{i=2}^{T} \tilde{\boldsymbol{m}}_{t_{i}}\right) \text {. }
$$

Putting (4) and (5) together, it is easy to see that the reference mesh is comprised of the selected pivot mesh, and a mean deformation field. In other words,

$$
\boldsymbol{m}_{\mathrm{ref}}=\boldsymbol{m}_{\mathrm{pivot}}+\frac{1}{T-1}\left(\sum_{i=2}^{T} \boldsymbol{u}_{t_{i}}\right) .
$$




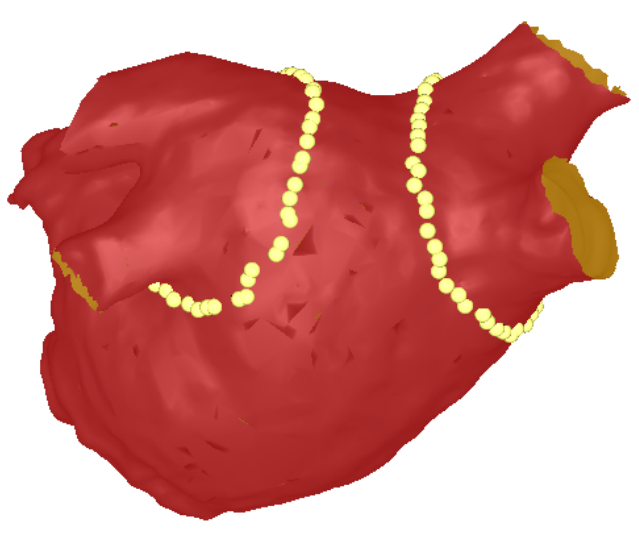

Fig. 3. Estimated reference model with associated mean pre-planned ablation lines (yellow). The mesh is seen from posterior direction. The reference model was derived from the template meshes by deformable registration.

The selection of a proper pivot mesh is important, because it determines the basic shape of the resulting mean mesh. This is why we carefully selected the pivot mesh, making sure that all relevant landmarks, namely the pulmonary veins and left atrium appendage, were clearly expressed. Figure 2 and Figure 3 depict the calculated reference model.

\subsection{Transfer of Planning Lines on Common Reference Model}

Planning lines $\boldsymbol{l}$ are represented as a set of points $\boldsymbol{l}=\left\{\boldsymbol{x}_{1}, \ldots, \boldsymbol{x}_{P}\right\}$ with $\boldsymbol{x} \in \mathbb{R}^{3}$. Each template mesh $\boldsymbol{m}_{t_{i}}$ has two planning lines $\boldsymbol{l}_{t_{i}, R}$ and $\boldsymbol{l}_{t_{i}, L}$ attached. They represent desirable ablation lines for right and left sided ipsilateral pulmonary veins, respectively. The planning lines are a subset of the corresponding template mesh, i.e., $\left\{\boldsymbol{l}_{t_{i}, R}, \boldsymbol{l}_{t_{i}, L}\right\} \subset \boldsymbol{m}_{t_{i}}$.

To transfer the planning lines from a template mesh onto the reference model, $\boldsymbol{m}_{\text {ref }}$ is registered to $\boldsymbol{m}_{t_{i}}$ using CPD. After registration, the two mesh models are optimally aligned based on the optimization criterion stated in (3). The planning lines $\left\{\boldsymbol{l}_{t_{i}, R}, \boldsymbol{l}_{t_{i}, L}\right\}$ are now projected onto the transformed reference model $\tilde{\boldsymbol{m}}_{t_{i}}$ with a nearest neighbor approach. By applying the inverse deformation field, the lines can be mapped onto the reference model $\boldsymbol{m}_{\text {ref. }}$. There, they are labeled $\hat{l}_{t_{i}, R}$ and $\hat{l}_{t_{i}, L}$, for right and left sided planning, respectively.

\subsection{Mean Planning Lines}

The mean planning lines are derived from the set of re-mapped ablation lines defined as

$$
\mathcal{L}=\left\{\hat{\boldsymbol{l}}_{t_{i}, R}, \hat{\boldsymbol{l}}_{t_{i}, L}\right\}_{i=1}^{T}
$$

Initially, each planning line consist of an arbitrary number of points. For consistency, each line $\boldsymbol{l}$ was interpolated with a cubic spline, and equidistantly sampled with a fixed number of sample points $P_{L}$. To investigate the spread and distribution among the pre-planned ablation lines, a common orientation and labeling was enforced. Each planning line represents a closed loop encircling the LA. The point closest to the top is defined as the starting point, and the remaining points are traversed in anterior direction.

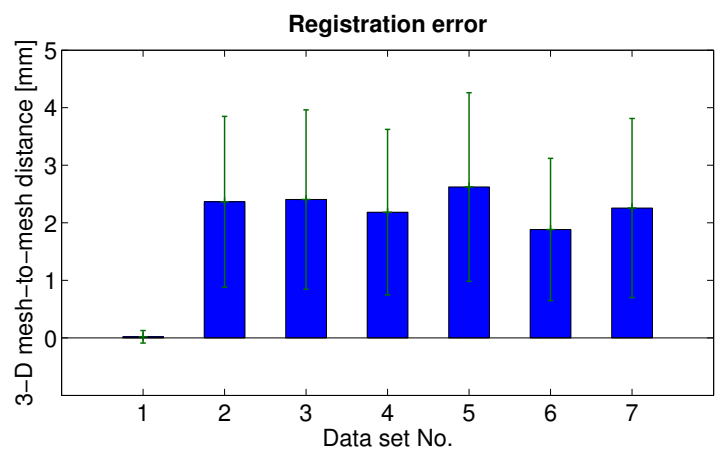

Fig. 4. Mean and standard deviation for residual mesh-to-mesh error after non-rigid registration of the reference model to the respective template mesh. Data set 1 was used as pivot mesh for generating the mean reference model.

After correct alignment of $\mathcal{L}$, mean reference lines $\boldsymbol{l}_{\text {ref }, R}$ and $l_{\text {ref }, L}$ are generated by averaging corresponding points along the interpolated lines. The set of re-mapped ablation lines visualized on the reference mesh is shown in Figure 2. The resulting mean lines are shown in Figure 3.

\section{EVALUATION AND RESULTS}

We evaluated our approach on $T=7$ LA meshes with attached planning lines. The pre-planned ablation lines were placed by an experienced clinician. The registration accuracy of the non-rigid registration of the reference model to the template meshes in terms of residual average mesh-to-mesh error is shown in Figure 4. The mean residual mesh-to-mesh error calculated over all meshes was $2.0 \mathrm{~mm}$. Data set 1 was selected as pivot mesh for the reference mesh generation. As can be seen in (6), the reference mesh is comprised of the pivot mesh with an additive deformation term. Hence, non-rigid registration of the reference mesh onto data set 1 is possible with a very low residual error, as illustrated in the first column of Figure 4.

The deviation of the re-mapped planning lines from their respective mean is evaluated as follows. For each point of the reference planning lines $\boldsymbol{l}_{\text {ref, } R}$ and $\boldsymbol{l}_{\text {ref, } L}$, the distance to the re-mapped planning lines $\hat{\boldsymbol{l}}_{t_{i}, R}, \hat{\boldsymbol{l}}_{t_{i}, L}$ with $i=1 \ldots T$ is calculated.

For further, more anatomically oriented evaluations, the planning lines were divided into eight equally spaced segments, as depicted in Figure 6. Based on this convention, we present the mean deviation of the individual planning lines per line segment in Figure 5. Line Segment 1 and 2 are located on the roof of the LA, Segment 3 and 4 are on the anterior side of the LA, 5 and 6 are below the inferior PV, and 7 and 8 are on the posterior side of the LA. Note that there is an additional pouch on the left side of the LA, the left atrial appendage (LAA). The LAA is located anterior the left-sided PVs, separated from them by a narrow ridge. The LAA runs on the left side along segments 2 to 5 .

The average distance of the re-mapped ablation lines to the reference ablation line was $2.9 \pm 1.9 \mathrm{~mm}$ and $1.8 \pm 1.5 \mathrm{~mm}$ for right and left sided planning lines, respectively. The maximum distance of a single planning point to the reference planning line was $8.5 \mathrm{~mm}$ and $7.6 \mathrm{~mm}$ for right and left side, respectively.

\section{CONCLUSIONS}

According to our analysis, the average deviation over all individual planning lines that were part of our date set was $2.4 \pm 1.8 \mathrm{~mm}$. 

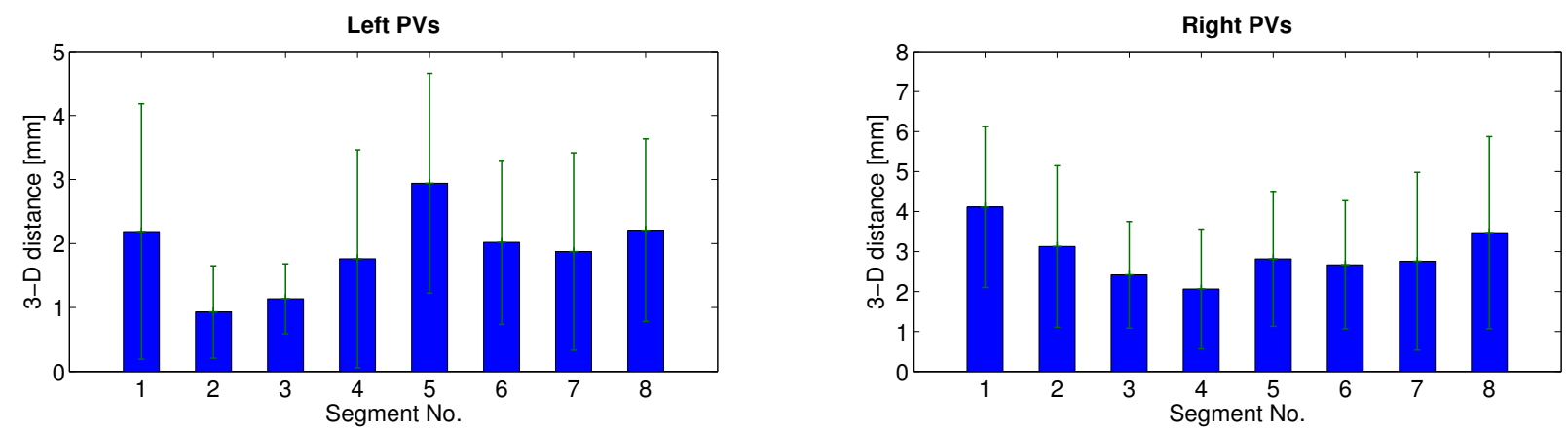

Fig. 5. Mean and standard deviation for the distance of pre-planned ablation lines per line segment. The left figure represents left sided planning lines, and the right figure right sided planning lines.

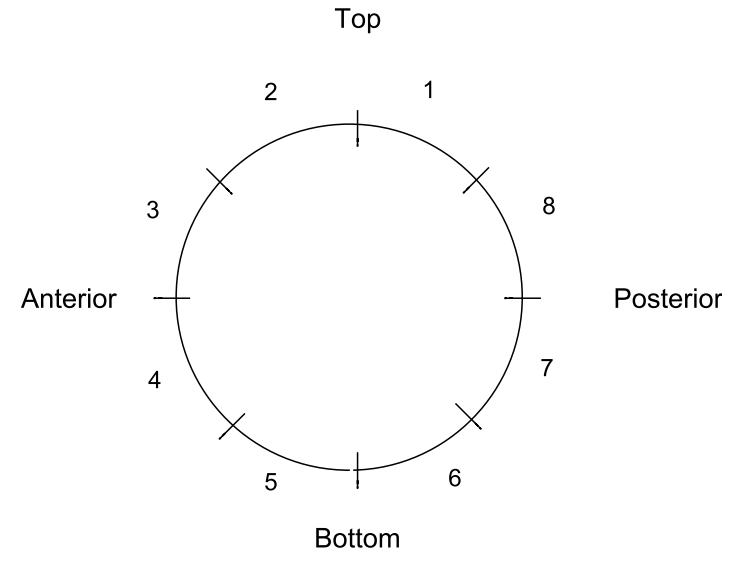

Fig. 6. Orientation and labeling of planning line segments. Line segment 1 and 2 are located on the roof of the LA, segment 3 and 4 are on the anterior side of the LA, 5 and 6 are below the inferior PV, and 7 and 8 are on the posterior side of the LA.

This results in a region for pre-planned ablation lines that is approximately twice this width. The smallest deviation was encountered in line segments 2 and 3 . We believe that this is due to the presence of the ridge between left atrial appendage and left PVs, that leaves limited space for setting up pre-planned ablation lines.

Based on feedback from physicians in this field, our findings appear plausible. In a nutshell, by comparing pre-planned ablation lines placed by an experienced clinician on LA models of actual patients, we found that one seems to have some $5 \mathrm{~mm}$ of 'wiggle room' despite the presence of prominent anatomical structures. After analyzing the inter-patient variance of manually placed planning lines and learning a general planning pattern, the design of an algorithm for fully automatic pre-planning of ablation lines will be the next step.

\section{ACKNOWLEDGEMENTS}

This work was supported by the German Federal Ministry of Education and Research (BMBF) in the context of the initiative Spitzencluster Medical Valley - Europäische Metropolregion Nürnberg, project grant Nos. 01EX1012A and 01EX1012E, respectively. Additional funding was provided by Siemens AG, Healthcare Sector. The concepts and information presented in this paper are based on research and are not commercially available.

\section{REFERENCES}

[1] Stijn De Buck, Frederik Maes, Joris Ector, Jan Bogaert, Steven Dymarkowski, Hein Heidbuchel, and Paul Suetens, "An augmented reality system for patient-specific guidance of cardiac catheter ablation procedures," IEEE Transactions on Medical Imaging, vol. 24, no. 11, pp. 1512 -1524, Nov. 2005.

[2] Martin Koch, Arne Langenkamp, Atilla Kiraly, Alexander Brost, Norbert Strobel, and Joachim Hornegger, "Navigation system with contact force assessment to guide pulmonary vein isolation procedures," in 23rd Conference of the Society for medical Innovation and Technology (SMIT), Tel Aviv, September 13 - 16, 2011.

[3] Felix Bourier, Dejan Vukajlovic, Alexander Brost, Joachim Hornegger, Norbert Strobel, and Klaus Kurzidim, "Pulmonary vein isolation supported by MRI-derived 3D-augmented biplane fluoroscopy: A feasibility study and a quantitative analysis of the accuracy of the technique," Journal of Cardiovascular Electrophysiology, 2012, [epub ahead of print].

[4] M. Hastenteufel, I. Wolf, C. Christoph, S. Yang, T. Boettger, M. Vetter, and HP Meinzer, "A novel method for planning and visualization of ablation lines for atrial fibrillation treatment," in Computers in Cardiology. IEEE, 2004, pp. 13-16.

[5] Alexander Brost, Felix Bourier, Andreas Kleinoeder, Jens Raab, Martin Koch, Marc Stamminger, Joachim Hornegger, Norbert Strobel, and Klaus Kurzidim, "AFiT - Atrial Fibrillation Ablation Planning Tool," in Vision, Modeling and Visualization (VMV), 2011, pp. 223-230.

[6] Roderick Tung, Eric Buch, and Kalyanam Shivkumar, "Catheter ablation of atrial fibrillation," Circulation, vol. 126, no. 2, pp. 223-229, 2012.

[7] Thomas Arentz, Reinhold Weber, Gerd Brkle, Claudia Herrera, Thomas Blum, Jochem Stockinger, Jan Minners, Franz Josef Neumann, and Dietrich Kalusche, "Small or large isolation areas around the pulmonary veins for the treatment of atrial fibrillation?," Circulation, vol. 115, no. 24, pp. 3057-3063, 2007.

[8] Martin Koch, Sebastian Bauer, Joachim Hornegger, and Norbert Strobel, "Towards deformable shape modeling of the left atrium using non-rigid coherent point drift registration," in Bildverarbeitung für die Medizin (BVM). 2013, Springer, [ahead of print].

[9] Andriy Myronenko, Xubo Song, and Miguel A. CarreiraPerpinan, "Non-rigid point set registration: Coherent point drift," Advances in Neural Information Processing Systems, vol. 19, pp. 1009-1016, 2007. 